\title{
Christoph Hubig:
}

\section{Ubiquitous Computing - Eine neue Herausforderung für die Medienethik}

\begin{abstract}
:
Ubiquitous Computing - A New Challenge for Media Ethics

We have to distinguish three types of media ethics: Applied ethics which focusses situations and raises normative questions depending on a particular situation; application-centered ethics, which supports or warns users, and the classical ethics of autonomy which gives new spheres for actions. For ethical challenges of UbiComp the third type is most important. UbiComp reduces the intentions of the user by decontextualizing the context of action. The actor is confronted with an "informed" reality. It is a problem, when there is no explicit delegation of services to the system and the media "clues" are disappearing and we don't see that reality is augmented. It is the first commandment of media ethics to show the clues of media via which it is possible to reconstruct the spheres which give possibilities for action. Concerning UbiComp, media ethics has to demand compensatory institutions like the concept of parallel communication, which allows for negotiating metacommunicatively on the communication processes delegated to smart systems.
\end{abstract}

\section{Agenda}

Angewandte Ethik, anwendungsbezogene Ethik und Ethik der Systemgestaltung ............................. 29

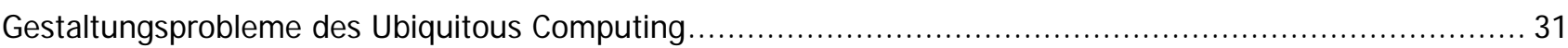

Medienethik als Ethik der Ermöglichung des Umgangs mit und in ubiquitären Systemen........................ 33

\section{Author:}

Prof. Dr. Christoph Hubig:

Universität Stuttgart, Seidenstraße 36, 70174 Stuttgart, Germany

胥 + 49 - 711 -6858 - 2491, $\square$ christoph.hubig@philo.uni-stuttgart.de, 매ttp://www.unistuttgart.de/philo/index. php?id=31

Relevant publications:

Die Kunst des Möglichen I - Technikphilosophie als Reflexion der Medialität. Bielefeld: Transcript Verlag 2006, 300 p.

Die Kunst des Möglichen II - Ethik der Technik als provisorische Moral. Bielefeld: Transcript Verlag 2007, 263 p. 
Überblickt man die Entwicklung praktischer Ethik in neuerer Zeit, so wird man feststellen, dass entsprechend der Ausdifferenzierung der Hochtechnologien sich auch die „Bindestrich-Ethiken“ differenzieren: Die Technik-Ethik, die man im weitesten Sinne auch als Medienethik begreifen kann, weil Technik insgesamt seit Francis Bacon ein Medium unserer theoretischen und praktischen Weltbezüge geworden ist (Hubig 2006), weist inzwischen Ausprägungen auf, z.B. als Nanoethik, Genethik, Ethik der Energiebereitstellung, Verkehrsethik, Umweltethik, „Kybernethik" oder Medienethik im engeren Sinne als Ethik der Informationstechnologien, die neue Möglichkeiten der Gestaltung des Umgangs mit Informationen bereitstellen. Mit Blick auf die IuK-Technologien finden wir Informationsethik, Computerethik, Netzethik, Kommunikationsethik u.v.a. mehr. Da solche Unterscheidungen im Wesentlichen an unterschiedlichen Gegenstandsbereichen orientiert sind, deren Gestaltung und Nutzung unterschiedliche normative Fragen aufweisen, werden die Übergänge fließend: Denn die einschlägigen und prominenten Probleme (z.B. Veränderungen der Arbeitswelt, Globalisierung und Virtualisierung individueller Kommunikation, Umgang mit Simulationen, Informatisierung der Handlungsumgebungen etc.) sind oftmals einem Zugriff geschuldet, der unterschiedliche Technologien gemeinsam in Anschlag bringt als "Converging Technologies", was seinerseits dadurch ermöglicht wird, dass diese Technologien zunehmend als Ermöglichungstechnologien unspezifisch werden bezüglich einer konkreten Zweckbindung oder einer Bindung an spezifische Problemlagen. Wäre mit Blick auf eine bestimmte Problemlage, z.B. das Gesundheitswesen und neue Therapieformen, eigens festzulegen, welche Argumentationslinien aus der Bioethik, der Genethik, der Nanoethik, der Wirtschafts- und Unternehmensethik (bezüglich der Rationalisierungsprobleme und der Rationierungsprobleme) sowie der Informationsethik und Kommunikationsethik (Schutz, Überwachung, Aufklärung, risikoadäquate Belastung der Prämienzahler etc.) in Betracht zu ziehen sind? Solche Fragen betreffen auch die Medienethik im engeren Sinne, wenn es um die neuen Systeme des Ubiquitous Computing geht, welches seinerseits je nach Akzentuierung der Entwicklungslinien Elemente des Mobile Computing oder Ambient Computing, des Pervasive Computing oder des Context aware Computing aufnimmt, umfasst oder unter diesen Titeln behandelt. Angesichts dieser verwirrenden Problemlage scheint mir zunächst eine kurze grundsätzliche Klärung des Anliegens praktischer Ethik angebracht.

\section{Angewandte Ethik, anwendungs- bezogene Ethik und Ethik der Systemgestaltung}

Worin liegt die Spezifik praktischer Ethik, wie sie sich in den „Bindestrich-Ethiken“ manifestiert? Ich schlage vor, drei Typen der Spezifizierung zu unterscheiden - hier für eine Medienethik i.e.S. -, die gemeinsam, aber in jeweils unterschiedlicher Weise, normative Probleme der Gestaltung und Nutzung von Elementen eines bestimmten Gegenstandsbereichs - hier: des Ubiquitous Computing - in einschlägigen Handlungsvollzügen betreffen.

Der erste Typ praktischer Ethik wäre eine Angewandte Ethik. Ob ich unter Nutzung eines Informations- und Kommunikationsmediums lügen darf (mit allen Konnotationen: täuschen, etwas vorgaukeln, etwas beschönigen, etwas stark vereinfachen, etwas unvollständig berichten, etwas unbegründet in Aussicht stellen, etwas verzerren, um Aufmerksamkeit zu erregen etc.) - in welcher Weise ist dies überhaupt eine spezifisch medienethische Frage? Hier kommt zum einen das in allen allgemeinen Ethiken, so unterschiedlich ihre Rechtfertigungsstrategie sein mag, prinzipielle Verbot des Lügens und der Täuschung zum Tragen. Allerdings wird auch in hoher Übereinstimmung konzidiert, dass "Ausnahmen die Regel bestätigen“, also in bestimmten Situationen Lügen zulässig sei, z.B. wenn es in einem unabdingbaren Interesse des Kommunikationspartners steht (etwa der Erzielung eines physischen oder psychischen Placebo-Effekts, seiner Sicherheit, des Erhalt seiner Lebenskraft und Motivation etc.) oder das Leben oder ein Minimum an Wohlfahrt eines Dritten gewährleistet, in diesem Sinne sogar kantisch wenn nicht als moralische, so doch als pragmatische „uneigentliche“ Pflicht erachtet werden kann. Der Umgang mit solchen Ausnahmen ist äußerst heikel; er macht das eigentliche Problem der Anwendung allgemeiner ethischer Direktiven aus. Er setzt ein umfassendes Wissen über mögliche Konsequenzen der Entscheidung sowie im Idealfall eine vollständige Erfassung der Problemlage voraus, auf deren Basis dann zu entscheiden ist, wie die „Anwendung“ allgemeiner ethischer Prinzipien qua Subsumtion des Einzelfalls in ihren Definitionsbereich von statten zu gehen hat, ohne den Sinn dieser Prinzipien zu unterlaufen oder zu verzerren und ohne durch strikte "formale" Befolgung eines Prinzips möglicherweise andere gegebenenfalls höher stehende Prinzipien zu verletzen; es wird ein Bezug analog dem "qualitativen Rechtsgehorsam" hergestellt. Die Spezifik einer solchen praktischen Ethik besteht in der von der Urteilskraft vollzogenen Bezugnahme allgemeiner 
ethischer Prinzipien auf Situationen. Das Surplus gegenüber einer allgemeinen Ethik ist dabei selbst kein ethisches Surplus, sondern eine gut begründete Beurteilung der Situation. Streng genommen wirft also die Frage, ob ich unter Nutzung eines Informationsmediums täuschen darf, kein spezifisch medienethisches Problem auf, sondern ein Beurteilungsproblem bezüglich der Situation. Dennoch sei weiter hier von einem ersten Typ spezifischer Medienethik die Rede. Es wird sich nämlich zeigen, dass gerade für das Ubiquitous Computing die Beurteilung und Modellierung von Situationen normative Fragen aufwirft.

Ein zweiter Typ der Spezifik einer Medienethik kann dahin gehend spezifiziert werden, dass eine solche Ethik als „Anwendungsbezogene" Ethik modelliert wird. Allgemeine Ethik wird hier nicht strikt angewandt, sondern es wird ein Bezug hergestellt zu möglichen (und für nicht-möglich erachteten) Anwendungen, über die die Gestalter und Nutzer der medialen Systeme disponieren. Eine solche Ethik erfasst mögliche Optionen der Gestaltung und Nutzung mit Blick auf mögliche Ziele und mögliche Mittel zu ihrer Verwirklichung. Sie entfaltet ein Tableau, vergleichbar mit einer Landkarte, auf deren Basis die Gestalter und Nutzer ihren Standort, mögliche Ziele und mögliche Wege zu deren Realisierung im Zuge von Mittel-Zweck-Verknüpfungen identifizieren können, wobei unterschiedliche normative Hypotheken des Gelingens der Zielrealisierung vorgestellt werden. Eine solche Spezifizierung der Ethik ist nicht rein analytisch-deskriptiv, denn sie fasst das Gelingen nicht bloß instrumentell-technisch auf, sondern diskutiert es im Lichte eines Gelingens überhaupt, also der Vorstellung, dass singuläre Vollzüge mit ihren Gratifikationen nicht das Streben insgesamt nach einem gelingenden Leben beschädigen. Das ist gemeint, wenn von „normativer Hypothek" der unterschiedlichen Handlungsoptionen die Rede ist, wobei auch unter der Konzession der Verschiedenheit einzelner Lebensentwürfe der Individuen darauf abgehoben wird, dass diese das Gesamtziel ihres Strebens nicht beschädigen. Es ist dies das Feld der Klugheitsethiken aristotelischer Provenienz, die in ihrem Liberalismus bezüglich der inhaltlichen Ausfüllung „des Guten“ dennoch harte Kriterien zu formulieren wissen, unter denen bestimmte Handlungsoptionen ausgrenzbar sind, sofern sie eben jenes Handlungsvermögen beeinträchtigen. Freilich bewegt sich eine solche Anwendungsbezogene Ethik im Modus von Ratschlägen, weil sie ihre Adressaten nicht nötigt oder verpflichtet, sondern allenfalls zu unterstützen oder zu warnen sucht unter der schwachen Unterstellung, dass diese Subjekte die erreichten Gratifikationen - insbesondere die Entlastung durch Delegation von Problemdiagnose und Problemlösung an die ubiquitären Systeme - später nicht bereuen.

Neben diesen beiden Typen spezifisch praktischer Ethik, die wir noch genauer im Feld der Medienethik angesichts der Herausforderung durch die Ubiquitous Computing-Technologien aufsuchen werden, lässt sich noch ein dritter Typ finden, der aus meiner Sicht der wichtigste ist, wenn es darum geht, die Spezifik praktischer Ethik gegenüber einer allgemeinen Ethik zu bestimmen. Großtechnische Systeme eröffnen uns neue Möglichkeitsräume des Handelns, sowohl im Sinne einer Erweiterung in räumlicher und zeitlicher Hinsicht für traditionale Vollzüge, die bisher an Grenzen oder Barrieren stießen, als auch im Sinne einer Neustrukturierung, die qualitativ andere, bisher nicht realisierbare Vollzüge erlaubt. Zugleich wird aber in der Regel auch für manche klassische Handlungstypen der Vollzug erschwert oder gar unmöglich; überkommende Handlungsweisen werden ersetzt, verdrängt, geraten in Vergessenheit (Hubig 2003). Dieser normale Wesenszug jeglicher Kulturentwicklung, der Kulturkritiker und Kulturpessimisten auf den Plan ruft, ist daraufhin zu reflektieren, ob mit dem Verlust von bestimmten Handlungsschemata nicht auch die einschlägigen Kompetenzen, die hierbei herausgebildet, fortgeschrieben und perfektioniert wurden, verloren gehen. Hier eröffnet sich, wie ich meine, ein genuin spezifisches Themenfeld für einen neuen Typ der Ethik, der freilich eine alte Wurzel hat: einer Ethik, die am Prinzip eines selbstbestimmten Handelns anhebt und als Pflicht formuliert, dass dieses selbstbestimmte Handeln sich nicht selbst aufheben, nicht mit sich selbst in Widerstreit geraten soll. Es ist die klassische Ethik der Autonomie, die prinzipiell auf die Vermeidung jeglicher Heteronomie als Zwang, der diese Grundfreiheit einschränkt, abhebt. Selbstverständlich begeben wir uns immerfort unter Herrschaft (die nicht mit Zwang verwechselt werden darf), sofern diese uns positive Handlungsfreiheiten gewährleistet - das Prinzip der Institutionalisierung. Die notwendigen Einschränkungen durch die Systeme, insbesondere die technischen Systeme, finden ihren Sinn darin, dass sie Handlungsoptionen eröffnen, die ohne sie nicht gegeben wären - also einen Beitrag zur positiven Freiheit leisten. Insofern ist, wie Max Weber herausgearbeitet hat, Herrschaft immer hypothetisch. Als "Chance, Gehorsam zu erzielen“, hängt sie davon ab, ob die von ihr versprochenen Gratifikationen willkommen oder die in Aussicht gestellten Sanktionen gefürchtet sind. Ein solches Anerkennungsverhältnis setzt aber eine Transparenz der Systeme voraus, aus der sich - wie wir sehen werden - wichtige Konsequenzen für eine 
Ethik der Systeme und hier im Speziellen: eine entsprechende Medienethik ableiten lassen. Grundrechte wie dasjenige der informationellen Selbstbestimmung heben auf diese Wahrung der Autonomie im Bereich der Informations- und Kommunikationstechniken ab. Eine solchermaßen gefasst „Ermöglichungsethik" macht den Kern einer Medienethik aus (wie auch analog z.B. einer Wirtschaftsethik, die sich mit Systemen der Arbeitgeber-ArbeitnehmerBeziehungen oder der Gestaltung von Handelsbeziehungen im globalen Markt befasst: $\mathrm{Ob}$ ich beim Handel betrügen darf, ist demgegenüber ein eher einfach zu klärendes Problem allgemeiner Ethik als angewandter Wirtschaftsethik). Für eine Medienethik angesichts der Entwicklung ubiquitärer Systeme ist daher unter diesem Typ praktischer Ethik zu fragen, inwieweit die Systeme basale Voraussetzungen des Handelns, nämlich die Identität der Subjekte und ihr bewusstes Entscheiden zu fördern oder einzuschränken vermögen. Die Akzeptabilität dieser Systeme, schwach gefasst als Akzeptanzfähigkeit, wäre eben nur dann gegeben, wenn die Anerkennung bestimmter Herrschaftsformen bewusst vollziehbar oder beendbar bleibt und so sowohl Gestalter wie Nutzer ihren Subjektstatus als gesichert erachten können. Dieser dritte Typ einer Medienethik als Ermöglichungsethik ist mit Blick auf das Ubiquitous Computing nun genauer zu verfolgen.

\section{Gestaltungsprobleme des Ubiquitous Computing}

Ubiquitäre Systeme heben darauf $a b$, unsere Handlungsumgebungen oder Elemente der Handlungsumgebungen in einem Sinne „smart" oder „intelligent" zu machen, damit sie die Fähigkeit zur Problemdiagnose und zur Problemlösung erlangen oder dem Nutzer ein Angebot zur Problemlösung machen (SFB 627, Bericht 2005/05). Dass wir den Vollzug von Teilschritten eines Problemlösungsprozesses (als technischem Handeln) an Apparate delegieren, ist nicht neu. Auch haben wir solche Teilschritte in kulturell verfestigten tradierten Schemata objektiviert, so dass bestimmte äußere Einrichtungen uns von der Aufgabe entlasten, Probleme zu identifizieren und eine Lösung zu suchen: Ein simpler Trampelpfad in unwegsamem Gelände, dem wir folgen können, gibt uns die Sicherheit, dass problematische Passagen vermieden und umgangen werden und er uns zu einem, beispielsweise durch einen Wegweiser indizierten Ziel „führt“. In metaphorischer Rede kann man davon sprechen, dass dieser Pfad "informiert“ ist (analog einem sachkundigen menschlichen Begleiter) und uns über bestimmte Verfasstheiten zu informieren vermag. Er kann als eine Institution im
Kleinen begriffen werden, die auf einer Bewährtheitstradition aufruht und Herrschaft ausübt, sofern man die Sanktionen des Herrschaftsentzugs fürchtet und auf die Gratifikationen der Herrschaft aus ist. Worin liegt der Unterschied zu ubiquitären Systemen?

Die smarten Dinge unserer Handlungsumgebung nehmen über ihre Sensorik Daten auf und bilden über die Sensordatenfusion ein Modell unseres Handlungskontexts. Es ist ein Beobachtungskontext, der sich von dem ursprünglichen Kontext dahin gehend unterscheidet, dass nur diejenigen seiner Wesenszüge in das Bild aufgenommen werden, die über die Sensorik erfassbar sind. Dieser Kontext ist also gleichsam in einem ersten Schritt „dekontextualisiert". Entsprechend den implementierten Strategien wird, angereichert durch Informationen, die aus dem Internet bezogen werden, dieser Kontext als so und so geartete Situation „interpretiert": Und entsprechend der jeweils identifizierten Problematik wird eine Situationsveränderung entweder angeboten oder gleich veranlasst. Es ist dies ein zweiter Schritt einer Dekontextualisierung, die nun einen Kontext erstellt, in dem das „Offensichtliche“ getan werden soll - "Context awareness".

Die Typisierung, die zu dieser Situation führt, kann auf zweierlei Weise vorgenommen werden: Erstens beruhen die systemischen Strategien, unter denen die Typisierung und anschließende Aktionen ausgelöst werden, auf seitens der Entwickler oder Anbieter vorausgesetzten Nutzerstereotypen als Adressatenprofilen, die wesentliche Merkmale des Kontext zusammenfassen bei unterstellten Nutzerpräferenzen, die ein Interesse an diesen Merkmalen begründen sollen. Darüber hinaus wird oftmals auf der Basis dieser Präferenzen auch abgeleitet, welches Interesse an Kooperationen mit anderen Präferenzenträgern oder einer Koordination der Präferenzenverfolgung gegeben ist. Zweitens können die Stereotype auch gewonnen werden durch ein adaptives Verhalten der Systeme, welche wiederkehrende Nutzungsansprüche als Routinen modellieren und dann entsprechend reagieren. Aktionen wie Einkaufen, Nutzung eines Verkehrsmittels in Verkehrssystemen, Accident-Management in Notlagen, Suche nach einem Zusammentreffen oder Vermeidung eines Zusammentreffens mit bestimmten Personen, Erhalt von zusätzlichen Informationen - auch aus der Vergangenheit - über Örtlichkeiten und Gesprächspartner etc. können auf diese Weise unterstützt und optimiert werden. Früher war zwar auch die Situation gegeben, dass der Handelnde sich Kontexten gegenüber sah, in denen kulturell verfestigte Strukturen und Schemata angetroffen wurden, die mit einer hypothetischen Zweckbindung verse- 
hen waren. Jedoch konnte sich der Handelnde zu innen jeweils in ein positives oder negatives Verhältnis setzen (zumindest im Prinzip). J etzt findet er sich in einer Handlungsumgebung wieder, in der die Dinge oder Ereignisse nach einer bereits herausgebildeten Zweckbindung prozessieren, die ein solches Sich-ins-Verhältnis-setzen zu ihr wenigstens erschwert oder in manchen Fällen gar unmöglich macht. Während der „klassisch“ Handelnde - die holzschnittartige Unterscheidung sei erlaubt - sich mit jedem Vollzug seine eigene Wirklichkeit schaffte und durch Erlebnisse des Misserfolgs und der Enttäuschung an der Widerständigkeit dieser Wirklichkeit oder an sich selbst im Weiteren arbeitete, sieht sich der Akteur in ubiquitären Systemen bereits einer verfertigten „informierten“ Wirklichkeit gegenüber. Dieser Effekt ist solange kein Problem, als er auf der Basis einer expliziten Delegation von Leistungen an das System zustande gekommen ist. Ubiquitäre Systeme haben aber nun gerade die Eigenschaft, diese Delegation zu erübrigen und die Entlastung auf eine Entlastung von der Delegation selbst auszuweiten. Das „Verschwinden der Computer" (Marc Weiser) findet hier seine Krönung, was durchaus willkommen sein kann in dem Sinne, dass eine Technik, die geräuschlos im Hintergrund ihren Job vollzieht, als die perfekteste erscheinen mag. Eine solchermaßen aufgewertete "Augmented Reality“ oder „Mixed Reality“ oder „Wirkliche Virtualität“ (Fleisch 2003) wird jedoch dann problematisch, wenn eine selbstbestimmte Nutzung der Systeme unter jeweils individuellen Interessen eingeschränkt wird.

Zunächst kann eine solche Einschränkung ersichtlich werden, wenn der Nutzer auf der Basis einer Irritation nicht mehr in der Lage ist, auf die Ursache dieser Irritation zurückzuschließen: systemische Strategien, die nicht adäquat erscheinen, oder Ergebnisse einer Koordination in Abhängigkeit vom Verhalten Dritter, die das System mit inm zusammen nutzen und ihre eigenen Präferenz verfolgen; oder eigenes Fehlverhalten im Umgang mit dem System; oder Selbsttäuschung über bisherige Handlungsroutinen, die das System registriert hat; oder die erst im Misserfolg bewusst werdende Einsicht über neue, abweichende Interessen (ein üblicher Effekt der Selbstvergewisserung über einen Interessenwandel angesichts einer Unzufriedenheit mit Ergebnissen, die bisher fraglos hingenommen wurden)? Irritationen eines zweiten Typs können entstehen, wenn die Nutzer - reibungsloses Funktionieren adäquater Dienste der Systeme seien vorausgesetzt - in Zweifel geraten, wer, was, wann und wo Informationen über eine spezifische Nutzung der Systeme aufnimmt, speichert oder weitergibt. Denn damit die
Systeme funktionieren, also ihre Leistungen im gesellschaftlichen Leistungstausch verortbar und die nötigen Investitionen amortisiert werden können, muss der Zugriff auf Systemleistungen explizit sein (Schutz des Anbieters), müssen andererseits Kontextinformationen dem Anbieter und dem Provider übermittelbar sein, damit die Systemleistung adäquat wird, muss drittens die Leistungsinanspruchnahme abrechenbar sein (Schutz des Providers), und es muss dennoch in dem gewünschten Maße die Privatheit des Nutzers gewährleistet bleiben. Aber nicht nur in dieser negativen Hinsicht ist Privatheit ein zu schützendes Gut vor Zugriffen, sondern, dem Prinzip der informationellen Selbstbestimmung folgend, gehört dazu, dass für den Nutzer die angebotenen Optionen oder die ausgelösten Prozesse als solche in einem Tableau oder einem Katalog möglicher Optionen transparent bleiben, damit dem Nutzer klar bleibt, ob dies die einzig möglichen sind, oder ob inm weitere Optionen vorenthalten werden. Angesichts eines vom System übermittelten Rates, sich so und so zu verhalten, ist dann die schlichte Frage, warum der Ratschlag erfolgt, nicht mehr einfach zu beantworten: Wird dies geraten, weil ich mich bisher in solchen Situationen üblicherweise so verhalten habe, oder weil das System bei unterstellten Präferenzen meinerseits ein Defizit oder eine Versorgungslücke identifiziert hat, oder weil ein Lenkungs- oder Koordinationseffekt im Interesse Dritter intendiert ist, oder weil sich Systemelemente amortisieren müssen, oder weil das System möglicherweise einseitig und unvollkommen informiert ist, oder weil das System nicht auf abweichendes Verhalten ausgelegt ist etc.? Selbst wenn die Systeme erlauben, entsprechende Datenspuren zu verwischen oder die Nutzung zu anonymisieren oder in bestimmten Kontexten die Nutzung zu verweigern, hinterlässt dies auch Spuren, aus denen Dritte Informationen ziehen können. Im Ganzen gesehen sind diese Probleme eines Informationsmanagements, an dem die Entwickler, die Anbieter, die Provider, die Nutzer und die Kontroll-, Überwachungs- und Haftungsträger beteiligt sind, wobei unterschiedliche Interessen gegeneinander stehen. Die medienethische Frage hierbei ist nicht primär, wie solche normativen Konflikte aufgelöst werden können oder sollen, sondern vielmehr, inwieweit die mediale Verfasstheit des Informationsmanagements in ubiquitären Systemen überhaupt die Möglichkeit eröffnet, solche Konflikte auszutragen. Es scheint hier der anfangs erwähnte dritte Typ praktischer Ethik besonders einschlägig zu werden für die Modellierung einer Medienethik, die auf Systeme des Ubiquitous Computing zu spezifizieren ist: Die Ermöglichungsethik, innerhalb derer dann Fragen des Anwendungsbezugs (welche Leistungen sollten 
unter welcher Informationspreisgabe an die Systeme delegiert werden) und schließlich Fragen der direkten Anwendung (wie sollen die Situationen typisiert werden, für die die Anwendungen greifen?) behandelt werden können.

\section{Medienethik als Ethik der Ermöglichung des Umgangs mit und in ubiquitären Systemen}

Für jegliche Medialität gilt, dass sie einen Möglichkeitsraum gibt für die Wahl von Mitteln und mithin die Realisierung von Zwecken. Dieser Möglichkeitsraum ist strukturiert, d.h. er weist unterschiedliche Eigenschaften in seinen Elementen und deren Binnenrelationen auf, die in unterschiedlicher Weise nutzbar sind. Im Zuge der Nutzung werden diese Elemente und Relationen als Mittel aktualisiert, und ihre Eigenschaften schreiben sich fort in die Gestalt der realisierten Zwecke, die auf diese Weise gegenüber ihrer geplanten Verfasstheit ein Surplus erhalten, das positiv oder negativ bewertbar ist und an dem sich die „Spuren“ der Medialität zeigen. Dies gilt auch für Informationsmedien im engeren Sinne, deren Möglichkeitsräume (z.B. die Verfasstheit der Kanäle) den Einsatz von Informationen zum Zweck der Kommunikation ermöglichen, begünstigen, erschweren oder verunmöglichen können. Nun sind in ubiquitären Systemen Informationsaufnahme, -austausch und -nutzung jedweder Art zu weiten Teilen an die Apparate delegiert, so dass metaphorisch davon gesprochen wird, dass "die Dinge kommunizieren und sich (selbsttätig) informieren“. Die medialen Voraussetzungen von der Sensorik über die Art des Datentransfers, die Strategien der Fusion und Modellierung, die Modi der Anreicherung durch Zusatzinformationen und die Algorithmen der Informationsweitergabe und Veranlassung weiterer „kommunikativer" Prozesse sind intransparent, so dass die Nutzer (und bisweilen die Entwickler) mit Ergebnissen konfrontiert sind, die als Zwecke für sich den Vergleich mit konkret intendierten Zwecken nicht mehr erlauben, weil die Ausgangsbasis lediglich noch allgemein unterstellte Präferenzprofile/Nutzerstereotypen sind. Aufgrund der fehlenden Erfahrung einer Differenz zwischen konkret intendierten und realisierten Zwecken entfällt die Einsicht in die Differenz zwischen beiden und somit die Erfassung von Spuren einer Medialität, über die sich allererst eine Einsicht in die medialen Voraussetzungen etablieren kann. Das bringt zwangsläufig Kompetenzverluste sowohl der Entwickler als auch der Nutzer mit sich, da sich ein Medium nur über seine Widerständigkeit als solches zeigt. Reale Möglichkei- ten sind immer nur durch Extrapolationen ex post erschließbar.

Beharren wir auf dem aus dem Autonomiepostulat abgeleiteten Prinzip der informationellen Selbstbestimmung, so wäre das oberste Gebot für eine Medienethik angesichts ubiquitärer Systeme, dafür zu sorgen, dass Medien Spuren hinterlassen, über die Rekonstruktionen der Möglichkeitsräume, in denen sich das Systemgeschehen abspielt, möglich werden. Erst dann könnte im Sinne einer Anwendungsbezogenen Ethik über Nutzensoptionen und im Sinne einer Angewandten Ethik über Situationstypisierungen normativ gestritten werden. Während im klassischen Modell des Handelns vorausgesetzt wird, dass wir uns bei der Wahl von Mitteln und der Wahl von Zwecken in ein bewusstes Verhältnis zu institutionellen Vorgaben setzen, auf die wir angewiesen sind, deren Angebote wir aber abzulehnen oder in modifizierender Weise zu nutzen vermögen, geht mit dem Einsatz ubiquitärer Systeme in gewisser Hinsicht eine Deinstitutionalisierung einher, solange die Schemata, auf denen das Systemgeschehen beruht, nicht über entsprechende Spuren rekonstruierbar sind. Angesichts der durchaus willkommenen Leistung, dass die Effekte ohne weiteres Zutun im Hintergrund gezeitigt werden („Heinzelmännchen-Effekt“) und einer geradezu willkommenen Entlastung gegenüber dem „klassischen“ Handeln, sich eben nicht mehr bei jeder Entscheidung zu entsprechenden institutionellen Schemata in ein Verhältnis setzen zu müssen, wäre zu fordern, dass die Spuren der Medialität auf andere Weise produziert werden müssen.

Wenn man also beides haben will - Entlastung und Beibehaltung einer Option, über die Spuren, die die Systemleistungen hinterlassen, sich über deren Medialität zu vergewissern -, ist eine kompensatorische Lösung erforderlich. Diese wäre dadurch erreichbar, dass neben der Mensch-SystemKommunikation, in der der Mensch seine Entlastung sucht, Ebenen einer Parallelkommunikation eingerichtet werden, in denen die Systeme ihre Spuren freilegen und in denen über die Medialität dieser Systeme normativ geurteilt werden kann bzw. entsprechende Urteile in einen Abgleich zu bringen wären. Prima facie bieten sich hierfür drei Ebenen einer Parallelkommunikation an.

Auf einer ersten Ebene der Parallelkommunikation wären zwischen Entwicklern, Dienstleitern, Providern und Nutzern - am besten mit Blick auf Pilotprojekte - die Architekturen der Sensorik, die Strategien der Bildung von Nutzerstereotypen, die Optionen abweichenden Verhaltens relativ zur Typik von Situationen, die Grenzen einer notwendigen Preisgabe von 
Privatheit und die Verfahren einer Transparenthaltung der Nutzeroptionen offenzulegen und dabei soweit zu optimieren, dass neue kulturelle Schemata einer „normalen“ Nutzung ersichtlich und damit Chancen und Risiken einer generellen Nutzung für die Adressaten disponibel werden.

Auf einer zweiten Ebenen wären in die Systeme Ebenen einer Parallelkommunikation einzuziehen bzw. vorzusehen für den Fall, dass im Verlauf der Nutzung Irritationen auftreten, sei es seitens der Nutzenden, sei es aber auch seitens der Systeme bezüglich der Frage, ob die in den Systemen modellierte Erwartungserwartung über die Nutzererwartung noch adäquat ist. In solchen Situationen der Irritation wäre on demand offenzulegen, unter welchen Informationen, gleich welcher Herkunft, unter welchen Strategien der Typisierung von Situationen und Problemlösungen die Systeme agieren bzw. für welche Verfasstheit der Nutzenden die Systemleistungen überhaupt adäquat sind. (Beispiel: Die Nutzung von Assistenzsystemen kann zu Kompetenzverlusten, etwa dem Sinken von Vigilanzschwellen, führen, die das System über seine Sensorik registriert und dann einen Dialog parallelkommunikativer Art über die Mensch-SystemKommunikation, die bisher stattgefunden hat, anbietet. Umgekehrt müsste der Nutzer in der Lage sein, bei irritierenden Systemleistungen deren Gründe zu erfahren, um dann über eine weitere Nutzung des Systems, eine modifizierte Nutzung oder einen punktuellen Ausstieg aus der Systemnutzung zu disponieren.) Erweitert auf andere und umfassende Bereiche würde eine solche Parallelkommunikation on demand während der MenschSystem-Kommunikation erlauben, sich in ein explizites Verhältnis zu der Systemverfasstheit zu setzen, wie es analog in den "hergebrachten“ Handlungsvollzügen gegenüber den diese tragenden institutionellen Schemata möglich war und ist.

Schließlich wären auf einer dritten Ebene über Monitoring und Diskursverfahren Foren einer gesellschaftlichen Parallelkommunikation zu etablieren, in der über Bewährtheitsstandards und in deren Lichte über Bewährtheit oder Misslichkeit der kollektiven Systemnutzung zu beraten ist. Solche Foren sind erforderlich, weil die individuelle und anonyme Systemnutzung, insbesondere aber auch die Effekte einer anonymen Vergemeinschaftung auf der Basis der Koordinationsleistungen der Systeme, nicht mehr erlauben, aus einer Beobachterperspektive die Verhältnisse der Einzelhandlungen zu den ehemals institutionalisierten Schemata und Strukturen zu registrieren, um von dort aus die Schemata zu modifizieren. Abweichungen, Nutzensverweigerungen, aber auch allgemein begrüßte Nutzungsstereo- typen würden auf diesen Foren explizit gemacht und damit Spuren der Medialität rekonstruiert, die im individuellen Handeln nicht mehr ersichtlich werden: Denn durch das Explizitmachen jener Umgangsweisen mit den Systemen wird zugleich explizit gemacht, inwieweit (a) die von den Subjekten intendierten Zwecke, (b) die in den Systemen als intendiert unterstellten Zwecke, (c) die Zwecke der Entwickler, Dienstleister und Provider mit den tatsächlich realisierten Effekten übereinstimmen oder sich hiervon unterscheiden.

Medienethische Erwägungen zielen also angesichts dieser Problemlage in den gegenwärtigen Diskussionen (1) auf die Forderung nach kompensatorischen Institutionen, in denen metakommunikativ über die an die smarten Dinge delegierten Kommunikationsprozesse verhandelt werden kann, (2) auf den Erhalt des Grundvermächtnisses der Selbstständigkeit und derjenigen Strukturen der Kompetenzbildung, die ihre Entwicklung gewährleisten, also den Erhalt von „Spuren“ der Aktionen informierter Umwelt, (3) auf die Wahrung höherstufiger Präferenzen (neben der direkten optimalen Präferenzerfüllung), die sich auf den Erhalt von Entscheidungsoptionen, weitest möglichen Handlungsspielräumen eines Sich-insVerhältnis-setzens zu Systemangeboten beziehen und (4) den Erhalt eines institutionellen Vertrauens, welches sich auf die Einhaltung von Regeln bezieht, die als solche gewusst werden und vergleichbar sein müssen mit ihrer Realisierung qua Befolgen durch Subjekte oder entsprechend eingerichtete Systeme, an die die Subjekte ihre Aktionen delegiert haben.

Dies kann insbesondere dadurch erzielt werden, dass die Systeme über ihre Aktionen und die Bedingungen, unter denen diese Aktionen stattfinden und vom System als funktional „erachtet“ werden, zu geeigneten Zeitpunkten auf jener zweiten parallelen Ebene Auskunft geben und damit explizit Spuren ihrer Medialität produzieren. Zur Sicherstellung einer intentionalen Nutzung der Systeme gehört, dass die denkbare und in bestimmten Bereichen mit konkreten Realisierungsoptionen versehene Aufhebung der Trennung von Online und Offline bewusst beschränkt wird auf diejenigen Bereiche, für die die Aufhebung dieser Trennung durch die jeweils in Interaktionen befindlichen Subjekte vorausgesetzt werden kann. Wenn das im Internet inzwischen verbreitete Agieren von Software-Agenten über eine entsprechende Sensorik in weite Bereiche der Handlungswirklichkeit der Subjekte implementiert wird, formt sich deren Wirklichkeit ohne ihr Zutun in einer Weise, die vielleicht bestimmte Handlungen als Einzelhandlungen optimal erfolgreich werden lässt, gegen den Optionswert des Handelns überhaupt aber verstoßen kann. Die Akzeptabilität von Ubi- 
Comp-Systemen wird in the long run davon abhängen, ob sie in ihrer Entlastungsfunktion den Optionswert des Handelnkönnens nicht verletzen, kurz: ihren Charakter als Medien trotz der von innen selbst vorgenommenen Formung der Wirklichkeit über kompensatorische höherstufige Architekturen zu erhalten vermögen. Dass wir - wie wir seit Marshall McLuhan (1968) wissen - jedes Medium insofern als "Message" begreifen müssen, als es in der Kommunikation genauso seine Spuren hinterlässt wie der Emittent einer Nachricht, bleibt nur solange wahr, als die Medien tatsächlich ihre Spuren hinterlassen. Entfällt dieser Effekt, so tritt der Verlust einer wie auch immer vermittelten Kontrolle ein, der unser Handeln dann nur noch zu einem bloßen Agieren werden lässt. Unser zunächst scheinbar „ausgefaltetes Gehirn“ (Negroponte 1995) würde dann von den Systemen dahin gehend zur Kenntnis genommen und registriert, dass auf diese Weise die Systeme ihr Eigenleben unter den Systemdirektiven perfektionieren können. Stammen diese Direktiven von Subjekten, die im Verborgenen zu bleiben suchen, ist dies noch der minder gravierende Fall. Verlieren diese Subjekte aber zauberlehrlingshaft selbst die Kontrolle über die Systemdynamik, wie man es bereits jetzt beim Handeln mit Derivaten und ihren höherstufigen Produkten im IT-gestützten Börsenhandel beobachten kann, geraten wir in eine kritische Situation. Für die menschliche Kompetenz und ihre Erhaltung bezüglich einer Interaktion mit Medien muss daher gefordert werden, dass die Vorfindlichkeit von Spuren des Medialen, an denen sich die Fähigkeiten der Subjekte abarbeiten und dadurch entwickeln, bewähren und fortschreiben können, gegeben sein muss. Dieses Gegebensein lässt sich medial organisieren über die erwähnten Prozesse von Metakommunikation und Transparenzbildung, für die die neuen Systeme auch ein Medium abgeben können.

\section{LITERATUR}

Fleisch, Elgar/Dierkes, Markus (2003): „Betriebswirtschaftliche Anwendungen des Ubiquitous Computing", in: Friedemann Mattern (Hg.), Total ver-

netzt. Szenarien einer informatisierten Welt, Heidelberg-New York: Springer, S. 145-157

Hubig, Christoph (2003): Selbstständige Nutzer oder verselbstständigte Medien. Die neue Qualität der Vernetzung", in: Total vernetzt. Szenarien einer informatisierten Welt, Heidelberg-New York:

Springer, S. 211-230
Hubig, Christoph/Heesen, Jessica/Siemoneit, Oliver/Wiegerling, Klaus (2005): Leben in einer vernetzten und informatisierten Welt. Context Awareness im Schnittfeld von Mobile und Ubiquitous Computing. SFB 627 "Nexus", Bericht Nr. 2005/05

Hubig, Christoph (2006): Die Kunst des Möglichen, Bd. I: Technikphilosophie als Reflexion der Medialität, Bielefeld: transcript

Hubig, Christoph (2007): Die Kunst des Möglichen, Bd. II: Ethik der Technik als provisorische Moral, Bielefeld: transcript

McLuhan, Marshall (1968): Die magischen Kanäle., Düsseldorf-Wien: Econ-Verlag

Negroponte, Nicholas (1995): Total digital. Die Welt zwischen 0 und 1 oder die Zukunft der Kommunikation. München: Hanser 